Cinémas

Revue d'études cinématographiques

Journal of Film Studies

\title{
Inédit : A. I. 28 [Attraction intellectuelle 1928]
}

\section{Sergueï M. Eisenstein}

Volume 11, numéro 2-3, printemps 2001

Eisenstein dans le texte

URI : https://id.erudit.org/iderudit/024850ar

DOI : https://doi.org/10.7202/024850ar

Aller au sommaire du numéro

Éditeur(s)

Cinémas

ISSN

1181-6945 (imprimé)

1705-6500 (numérique)

Découvrir la revue

Citer cet article

Eisenstein, S. M. (2001). Inédit : A. I. 28 [Attraction intellectuelle 1928]. Cinémas, 11(2-3), 147-160. https://doi.org/10.7202/024850ar d'utilisation que vous pouvez consulter en ligne.

https://apropos.erudit.org/fr/usagers/politique-dutilisation/ 


\section{INÉDIT \\ A. I. 28 [Attraction intellectuelle $1928^{1}$ ]}

\section{Sergueï M. Eisenstein}

Ce n'est pas encore un essai.

$\mathrm{Ni}$, dieu nous en garde, un traité. Pour l'instant, ce n'est qu'un faisceau de réflexions.

De réflexions hérissées.

Qui partent dans tous les sens.

Qui n'ont pas encore été canalisées dans une seule direction.

En mathématiques, il se trouve que:

1. Dans une infinité de points, il doit exister un repère géométrique de points, reliés de manière à ce que le produit des distances de chaque point à deux autres points constants $F$ et $F 1$ (foyers) soit une grandeur constante égale à a2.

2. Par l'analyse des conditions spécifiques de la position de ces points et en utilisant le système des coordonnées, on peut en déduire qu'ils sont regroupés dans une solution formulée [en une formule abstraite], répondant à tous les points ensemble et plus exactement à chaque donnée en particulier.

Algébriquement, cela donne une équation qui vaut pour tous les points de même propriété. En même temps, cela désigne la courbe qui les relie entre eux.

Dans ce cas: $\sqrt{ }(x-c) 2+y 2 \cdot \sqrt{ }(x+c) 2+y 2=a 2$

3. Ensuite, à nouveau par des méthodes originales, le concept abstrait exprimé par cette formule sera traduit de façon perceptible, en un dessin dans l'espace.

Ce dessin qui permet de percevoir concrètement cette formulation est réalisé avec les points dont il a été question plus haut. 
Dans ce cas, le dessin sera une cassinoïdale (les ovales de Cas$\operatorname{sini):~}$

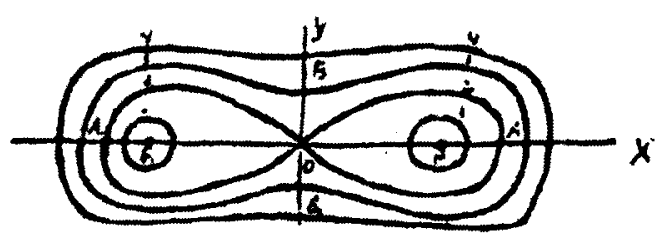

Il s'agit du Cassini à propos duquel Bergson raconte une anecdote:

... remarque d'une dame, que l'astronome Cassini a invitée à voir une éclipse de lune mais qui est arrivée en retard.

- Monsieur Cassini sera assez bon pour recommencer depuis le début, fit-elle remarquer ${ }^{2}$.

Avec d'autres conditions, cela donnerait une lemniscate de Bernoulli, une strophoïde, une sinusoïde, une ellipse, un cercle et ainsi de suite à l'infini.

C'est seulement traduites dans l'espace que ces formulations « abstraites" nous apparaissent tout à fait claires, c'est-à-dire que l'on peut les percevoir physiquement et qu'elles rendent compréhensibles les points d'un quelconque repère géométrique idéal de points.

Il en va de même pour l'analyse sociale:

1. Une masse de faits, déterminés par les mêmes conditions socio-économiques.

2. L'analyse marxiste, apportant une solution tactique par rapport à ce système de faits, est pratiquement pertinente pour chaque fait pris séparément.

L'analyse formulée en une résolution, un slogan.

Slogan ${ }^{3}$ — signifie: «solution» en allemand.

Résolution ${ }^{4}$ — signifie: "solution" en français.

Une solution qui existe en tant qu'appréciation de faits passés depuis longtemps et qui intervient en tant que directive dans les conditions actuelles.

Et troisièmement - de la même manière, elle doit devenir perceptible physiquement par la masse pour laquelle elle a été forgée. 


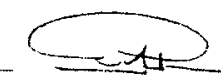

Et au lieu de cela, on a fait appel à ces piteux vestiges de l'esprit, qui ont été laissés pour plus tard sous l'enseigne d'art. Art aux trois quarts déjà tombé de son propre cadre sous la pression de l'essor de la science. Art qui, aux trois quarts déjà, passe certes peut-être pour une pseudo-science mais pour une science tout de même.

Et sur ce point, comme dans le jeu, il faut crier "Tu brûles!».

Un slogan pour la masse!

Tel est le mot d'ordre, le cri, la revendication dans les pages de la Komsomolskaïa Pravda.

Un objectif de lutte pour les laboratoires de recherche.

Le troisième point coince:

La matérialisation du slogan.

Et ici nous nous trouvons au brusque tournant d'une approche absolument nouvelle.

"Oui, oui, l'art doit toujours apporter quelque chose de nouveau ", m'a dit, dans le Kholme d'autrefois (province de Pskov), une certaine Maria Timofeevna, ingénieur militaire.

Coiffée à la Lysistrata et versant de la mélasse (en 1919) dans son thé aqueux.

Ce n'est pas grâce à Maria Timofeevna et à sa confiture.

Mais parce que l'évolution de la technique et le perfectionnement des formes sociales vont à un rythme tel, qu'il est temps de réclamer à pleine voix, au lieu des formes d'exaltation de semifanatiques ${ }^{5}$ que procure la salle de spectacle, une approche plus industrielle et plus rationalisée de cet objectif capital.

Ça suffit de plonger au fond de la mer à la recherche de perles.

Au risque d'être bouffé par les requins. On ne peut ranger la profession de "chercheur de perles" dans aucun syndicat. Si ce n'est celui d' "ouvrier de l'alimentaire", dans la mesure où on court le risque d'être bouffé?... L'époque des arias de Nadir est bonne pour les archives.

"L'aria de Nadir» signifie tout autre chose aujourd'hui!

"En retournant le tas de fumier..."

On peut avec satisfaction être coq et pour un grain de perle retourner tout le tas de fumier.

Le tas de fumier du sujet, de la fable, de l'intrigue, de la galerie des images, etc. 
Afin d'y déterrer, de la main rusée de l'«auteur », le grain enfoui du slogan.

Nous ne voulons nullement faire les cancres avec Plekhanov, mais dans la question de la "connaissance et l'art", par «image", nous ne voulons aucunement comprendre plus longtemps $L a$ Pauvre Lise ou Le Brave Soldat Chveik ${ }^{6}$.

Les ovales de Cassini correspondent mieux à notre âge.

La géométrie analytique nous est plus proche.

Nous exigeons la mise en forme spatiale immédiate de l'essence du slogan, exactement de la même manière que l'on transpose sans délai son dessin avec des lettres découpées dans du contreplaqué et tenues au bout de perches par les masses qui manifestent.

De la même manière la compréhension que nous en avons se transforme instantanément grâce aux distiques de nos auteurs de slogans!

Mais ces auteurs préferent parodier Thésée.

En se trouvant aux portes largement ouvertes du slogan, c'est-àdire qui se sont ouvertes depuis la première ligne dont il a pris conscience, que fait-il: il attache à la poignée de cette porte le bout du fil et s'avance vers l'autre bout du labyrinthe, par lequel on arrive à cette porte ouverte. Le labyrinthe reproduisant le chemin empirique à travers les phénomènes réels qui conduisent de toute façon, "d'une manière ou d'une autre", au slogan de ce genre.

Avec Thésée, c'était au moins motivé: il y allait pour trucider le Minotaure.

L'auteur, pénétrant au plus profond du labyrinthe logique, se hisse dehors par le toit, fait descendre dans le trou le pauvre récepteur et lui donne dans la main l'autre bout du fil.

Se tenant à ce bout, le pauvre homme doit gagner la porte de sortie par le même labyrinthe logique du sujet tout tracé.

Et... enfoncer la porte ouverte.

Comme dans: «Au commencement était le Verbe».

Car la solution existait depuis le tout début.

L'acte de "l'œuvre d'art" revient à masquer dès le début une tendance déjà donnée.

Cette voie correspond aussi à celle des mathématiques.

Les démonstrations de théorèmes. Bien que vous le connaissiez, vous devez le démontrer. 
Ou bien (plus évident) la solution d'un problème dans un manuel, quand on a la réponse à la dernière page.

Mais ici, il y a une motivation. Ces "jeux" enseignent la méthode pour découvrir les solutions, cette activité sert moins que tout à introduire dans la conscience la "réponse " et la solution.

La coïncidence terminologique est elle aussi caractéristique.

Lorsqu'un dramaturge accumule situations sur situations pour la description d'un "type " (voilà encore une "résolution" pour toute une couche de la population!), il est très intéressant de noter ce que l'on dit alors: il "donne naissance" au type du misanthrope, de l'avare, etc.

De la même manière que l'on fait éclore des poussins. $\mathrm{Ou}$ que l'on fait éclore des formules algébriques.

Et alors, qui a besoin de cette incubation, de cette "éclosion", quand c'est la conclusion qui importe?

C'est comme si l'on se déplaçait à pied à New York pour vérifier l'exactitude de la signature sur une lettre de change alors qu'on peut recevoir l'information immédiatement par radio.

Nous vivons de manière condensée.

Aux États-Unis par exemple, le premier niveau de condensation, c'est la bande des livres avec un résumé du contenu; au deuxième niveau, la bande ne propose déjà plus le contenu littéraire du livre mais annonce qu'on peut le lire en 3 heures 45 minutes.

Voilà ce qu'on exige pour transmettre de façon sensible les caractéristiques d'une ouvre.

Sur la question du Misanthrope et d'Harpagon, nous pouvons être assertifs jusqu'au bout.

La méthode d'“éclosion des personnages à travers une série d'actions " (des péripéties en 5 actes) est récusée, au moins dans la seule sphère dont nous nous préoccupons : le cinéma.

Ici, le mot décisif est celui de type.

Qu'est-ce qu'un type véritable?

C'est la manifestation la plus évidente du dessin (y compris, bien sûr, les expressions du mouvement) d'un homme, de toute la complexité de son comportement (behaviour*) social et individuel.

* Mots, groupe de mots ou expression apparaissant en allemand, anglais, français ou latin dans le texte original russe. 
Le type, c'est un hiéroglyphe socio-biologique, mais tel que chacun sera son propre Champollion par rapport à lui!

Comme exemple d'un type à cent pour cent social, on peut prendre les mencheviks dans Octobre, et avant tout, bien sûr, celui qui sans arrêt et bêtement levait son doigt, que tous gardent en mémoire.

En contemplant le "typage" vous défaites mentalement l'écheveau des différentes prémices qui l'ont constitué.

Cela signifie que le type est diamétralement opposé à l'acteur.

L'acteur - neutre dans l'idéal - de l'impersonnel "Bureau de location d'acteurs, carte $N^{7}$... " est devenu un Don Diego ${ }^{8}$, à travers une série d'actions exécutées successivement de manière concrète.

C'est-à-dire en rembobinant l'écheveau des actions en une pelote qui constitue le "personnage».

Le typage est diamétralement opposé à la mécanique de la présentation et de la réception.

Ils sont diamétralement opposés par les époques qu'ils représentent.

Voilà pourquoi il est mauvais d'ajouter au type des situations qui lui donnent une caractérisation.

Ainsi était-il pénible de voir dans La Mère la scène où le brillant type du père, éloquent par son aspect extérieur comme le "Cantique des cantiques" de Salomon, tout à coup, en sus, "caractérisé " par une série d'actions.

Tu as montré quelqu'un de si remarquable, tu peux le faire disparaître.

Ne le force pas à jouer.

Sinon, on appelle ça de l'huile huileuse.

Dans La Fin de Saint-Pétersbourg, il y a un progrès remarquable qui a été fait de ce côté-là.

Et donc changement dans la perception. Et donc, plus exactement, en s'adressant à d'autres fonctions de la perception (c'està-dire la réaction).

En premier lieu, [en s'adressant] au remplissage par un matériau qui provient des événements montrés (accumulation de stimuli).

En deuxième lieu, en introduisant des séries associatives tirées du bagage de celui qui perçoit [le spectateur]. 


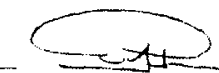

Il est tout à fait clair que la première étape correspond à un stade culturel considérablement plus fruste que la deuxième, qui suppose un bagage d'images associatives, une certaine "culture" (comprenez le jeu de mots $^{1}{ }^{9}$ ).

Je pense qu'il y a entre eux des intervalles capitaux, des correspondances comme entre le stade de l'économie naturelle et ceux qui suivent.
Au stade de l'économie naturelle, la langue ne connaît pas de mots abstraits. Car alors il n'y a pas encore de qualités qui soient indépendantes des choses: la bonté, c'est un homme bon; l'amour, c'est Untel qui aime Madame Untel; la fatigue, c'est celle d'un homme.
(Ioffé, Culture et style ${ }^{10}$ ).

Le stade des concepts, détachés des situations concrètes [est à venir].

La façon dont résonne "J'aime» est déjà un stimulus conditionné. Mais "l'amour" n'est pas encore relié à la chaîne des réflexes conditionnés.

Autre exemple: l'algèbre qui, au début, ne connaissait pas encore les nombres concrets.

Indiquons une différence qui existe entre le cours de géométrie actuel et son origine première. Dans les cours d'aujourd'hui, c'est le domaine de la science du nombre et de la science de l'espace qui a le plus d'importance. En mesurant des distances géométriques, on les exprime à l'aide des nombres et ainsi on a la possibilité d'utiliser l'algèbre pour tirer des conclusions sur les différentes relations entre ces grandeurs géométriques. C'est de cette manière que l'on tire les conclusions de base sur le rapport entre les côtés d'un triangle. Le théorème de PYTHAGORE, en particulier,

$(\mathrm{BC}) 2=(\mathrm{AB}) 2+(\mathrm{AC}) 2$

est compris parfois ainsi: le carré du nombre qui équivaut à la mesure de la longueur BC est égal à la somme des carrés des nombres qui équivalent à la mesure des deux autres côtés. Les Grecs anciens ne pouvaient faire une telle utilisation des nombres en géométrie, car leur connaissance de ceux-ci se limitait aux nombres rationnels, tout à fait insuffisants dans la mesure 
des grandeurs. Ce même théorème de Pythagore nous aidera très facilement à nous en convaincre: prenons en effet le triangle rectangle isocèle dans lequel

$\mathrm{AB}=\mathrm{AC}=1$ (c'est-à-dire l'unité de longueur); alors:

(BC) $2=2$

et le mathématicien moderne dira donc aujourd'hui que la mesure du côté $\mathrm{BC}$ est égale à $\sqrt{2}$. Les Grecs anciens ne pouvaient le dire, tout simplement parce qu'ils ne connaissaient pas un tel nombre. En conséquence, il y avait à leurs yeux des grandeurs qui ne s'exprimaient par aucun nombre connu d'eux. Il n'y a pas si longtemps que l'on en est arrivé à cette conclusion ; il ne nous sera maintenant pas difficile de comprendre qu'elle était déjà connue de l'école des pythagoriciens. L'existence de grandeurs incommensurables frappait les Anciens: on rapporte que le membre de la confrérie des pythagoriciens qui osa annoncer aux non-initiés cette dysharmonie du monde a subi un châtiment cruel de la part des dieux. En même temps, cette découverte a ouvert les yeux des savants anciens sur l'inévitable fossé entre la grandeur et le nombre, et c'est pourquoi la science des nombres était inapplicable à l'étude des grandeurs. D'où le côté inhabituel de l'argumentation d'Euclide, qui revêt un caractère purement géométrique: il n'y a pas ce qu'aujourd'hui on appelle l'application de l'algèbre à la géométrie. Le théorème de Pythagore, par exemple, était considéré infailliblement ainsi: le carré de l'hypoténuse est égal à la somme des carrés des cathètes.

De plus, comme la grandeur géométrique était plus largement connue que le nombre, les principes de l'algèbre ont ainsi été exposés sous une forme géométrique, comme dans l'équation bien connue:

$$
(a+b) 2=a 2+2 a b+b 2
$$

que l'on énonçait ainsi: le carré de la somme de 2 segments est égal à la somme des carrés de chaque segment plus le double du quadrilatère, produit des segments donnés.

Et donc, l'effet produit par le principe de l'insertion de séries associatives (se manifestant de manière démarquée du mécanisme fondamental de la réflexologie). 


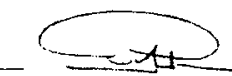

Est-ce que dans ce domaine il y a chez nous une quelconque pratique (des résultats)? Y a-t-il quelques premiers jalons-bornes sur ce chemin?

Il y en a, et plus qu'il nous semble.

Mais nous n'avons tout simplement pas envisagé la série de ces phénomènes de cette manière, sous un tel angle.

Le signe distinctif de A. I. 28, c'est son caractère hiéroglyphique.

Sa marque fixe, qu'on le veuille ou non - "symbolisant" signifiant conventionnellement une sorte de processus c'està-dire engraved by* [gravé par] un processus dynamique.

Ci-dessus nous avons envisagé un des exemples primaires et des plus grossiers (évidemment pour la transition) de ce cas, celui du typage, qui se trouve à la charnière entre le cinéma du passé et de l'avenir.

Le processus d'intégration de la chaîne des associations behaviouristes est déchiffré. Le type est un hiéroglyphe sociobiologique.

Avec le type, ce sont les premiers bourgeons du nouveau ci-

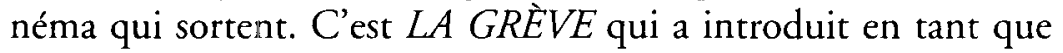
procédé le typage d'un épisode - parfois minimal - en gros plan (types de laquais, de mouchards, etc.).

Ensuite 1905. Pierre Ir, de nuit, arrosé d'eau et de la lumière des projecteurs. C'est de lui que vient la "mode» des monuments. Pierre Ir sur son cheval : cette photo a fait le tour de la presse.

Sur pellicule, roulé en bobine, il a fini à la corbeille. Le sort a voulu que La Cinquième [année] soit limitée au Cuirassé. Pierre a laissé derrière lui la mode des monuments mouillés et, avec les lions, a poussé un rugissement sur les escaliers d'Odessa ${ }^{11}$.

C'est la passion maniaque pour les statues qui a commencé. Après le "lion", les statues ont été filmées par tout le monde. De toutes les façons. Avec insistance et opiniâtreté.

De manière instinctivement juste. Bien sûr, pas gratuitement.

La statue, c'est un pas de plus vers la sphère du nouveau rapport [à l'objet].

La statue, c'est le pas qui vient après le type.

Pour la première fois elle triomphe, en "jouant" un être vivant: un effet de montage, avec le cupidon qui tourne dans le 
théâtre d'Odessa qui remplace par le "mouvement " de la figure, la main tendue, le vol de l'obus, et l'effet de montage des lions de marbre "qui se lèvent brusquement» à Anapa avec l'explosion des obus à Odessa. Les statues "ont joué", comme les gens.

En se positionnant de la sorte, comme la suite logique des gens qui jouent ou sont des types, la statue commence fonctionnellement à se complexifier. Soit elle commence alors à devenir un calembour d'homme vivant (Kerenski et Napoléon; "la Maternité" et les soldates du bataillon féminin; une vieille du bataillon et «le Printemps", c'est-à-dire un parallélisme encore purement spatial: la même pose pour Kerenski et Napoléon, le contraste brutal entre "le Printemps» qui se donne et la soldate mal fagotée ${ }^{12}$. Pour «la Maternité» et les soldates, la relation est déjà moins directe... Enfin la statue de la maternité se présentant comme calembour d'une expression injurieuse donnée par l'inscription ${ }^{13}$, ne relève déjà plus d'un parallélisme immédiat mais est entièrement au sommet des réflexes conditionnés par identité "formelle" du réflexe de base à [l'idée ou l'image de] la "maternité ". En faisant appel à un stimulus inhabituel pour l'idée de maternité (il est naturel que par réflexe le stimulus lié à l'«expression injurieuse» provoque en premier lieu la première moitié - ici la partie omise - d'une expression à deux termes bien connue ${ }^{14}$ ), il en résulte une association directe inattendue et par conséquent un effet comique.

Ensuite le travail de la statue est entièrement déplacé dans la sphère du jeu.

Sur le plan purement physique, le groupe des statues (3 lions) a créé par le biais du montage la "phase du mouvement» (un saut); maintenant, un groupe de 15 idoles, également par le montage, tente de déterminer la "phase de l'idée". Il développe une idée. («Disqualification» de la divinité ${ }^{15}$ ).

N.B. Remarquez qu'elle ne représente pas la phase de l'idée comme dans Le Dernier des hommes ${ }^{16}$ et autres abominations; elle conduit la phase de l'idée, comme une sorte de chaîne strictement logique de raisonnements (argumentations). Cela aurait dû se trouver avant dans Der letzte Mann*, comme Léger* a commencé par dessiner des machines.

Dans tous les cas, ce chemin conduit ce qu'on appelle notre art à une étape considérablement plus élaborée. 


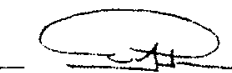

C'est justement dans cette direction qu'il faut mener les recherches.

Au lieu de quoi nous faisons maintenant une chose horrible.

Nous faisons l'essai d'une brillante méthode analytique en qualité de méthode constructive.

Ayant une connaissance analytique de l'art en tant que superstructure, nous gaspillons des wagons d'énergie à la construction consciente par avance de telles superstructures, qui conviennent au jour d'aujourd'hui.

N'ayant pas, en tant que contemporains et participants, de perspective suffisamment claire (qui exige ne serait-ce qu'un peu de recul) sur le «jeu » des forces productives du moment, qui de plus varie de manière dynamique - la superstructure n'est imaginable que par la résultante du jeu de ces distorsions -, il y a peu d'espoir dans la construction consciente de cette superstructure.

Pire que cela, la véritable superstructure fait maintenant quelque chose comme perforer l'intestin de cette superstructure en serre [artificielle].

$\grave{A}$ un moment ou à un autre elle va bien finir par surgir.

Elle finira bien par détruire cette chose imaginée de l'intérieur.

N'est-ce pas le sort d' "OCTOBRE»?

Quand toute la série des points de vue de demain (peut-être déjà d'aujourd'hui au soir ou de cet après-midi...) détruit le bonheur de l'intégrité d'hier.

Nous avons omis une autre circonstance: ce que nous savons sur l'art en tant que superstructure sur le plan de sa construction en serre ne nous aide en rien, elle sera construite sous une forme ou sous une autre en se passant de cela (sans qu'on s'en mêle particulièrement), peut-être pas dans l'art mais dans une quelconque autre branche de la culture.

C'est même certain.

Cette prise de conscience est intéressante par un autre aspect.

Car la prise de conscience du mécanisme de création l'élimine en tant que création.

La création consciente est un travail scientifique ou du moins rationalisé. 
Et ce travail doit être dirigé vers l'élaboration et la mise au point de ce jeu de déductions qui naît de l'écume et qui remplace le jeu des passions vivantes du récepteur.

Cela tombera au fond du gouffre sans espoir avec le théâtre dont il est la base (voir $L E F, 1923, \mathrm{n}^{\circ} 3$ - «Le montage des attractions »: "...Le chemin de la connaissance, au travers du jeu vivant des passions du récepteur"). "C'est spécifique au théâtre..." et c'est de là que pendant quelques trente petites années il a été pris en location pour le cinématographe.

Ici pour la première fois passe un Rubicon décisif et de principe entre le théâtre et le cinéma. Une fois pour toutes.

Le principe de l'attraction est indestructible.

Qu'il soit émotionnel ou intellectuel, sa visée sociale est la même: le labourage idéologique.

Nous apportons les corrections de l'année 28 sur l'attraction.

L'attraction théâtrale sur le «sentiment».

L'attraction cinématographique sur la "conscience".

Ça c'est pour la réflexion de cuisine.

Pour parler sérieusement:

Attraction 1923. Sensuelle

Le stimulus du réflexe non conditionné ayant une action directe.

Sphère de travail: - LE THÉÂTRE (et les 30 premières années du cinéma y compris Potemkinet et

l'Octobre à 33\%)

$$
\text { †sauf les lions*!!! }
$$

Attraction 1928. Intellectuelle.

Base de l'action sur la chaîne des réflexes combinés. action par chaines associatives.

Sphère de travail: -LE CINÉMA

VÉRITABLE.

(33\% - Ligne générale et $66 \%$ de l'Octobre)*

N.B. Merkwürdig* [remarquable] : cette même position (2e) a été prise de nos jours (1924) lorsqu'il a fallu établir pourquoi l'action au cinéma doit obligatoirement être filmée en vue du 
montage. L'argumentation en a été faite, pour la partie "du montage des attractions », dans le livre médiocrissime de Belenson, Le Cinéma aujourd'hui ${ }^{17}$. Il a fallu 4 ans pour faire un tour d'horizon des possibilités de cet ausschlaggebendes Prinzip (Eigentumlichkeit)* [principe décisif (originalité, particularité)].

5. IV. 1928

\section{[NOTES DE SERGUEİ EISENSTEIN]}

I. N.B. Les réactions inconditionnelles réflexes ne sont nullement exclues du typage: la sensation tout à fait behaviour ${ }^{*}$-iste de l'objet de contemplation propose dans tous les cas une sorte d'identification physique avec les éléments les plus nettement exprimés du behaviour-comportement contemplé, la seule capable de donner une "déduction" physique selon l'ancienne terminologie psychique: une appréciation abstraite.

II. N.B. Pour plus de clarté, le raisonnement se fera dans la [terminologie] de convention, équivoque, utilisée par tous, et non purement réflexologique, mais dans une acception purement réflexologique des termes. Il s'agit à nouveau de l'exposition de la "substitution", comme en mathématique, quand on écrit x pour $\sqrt{2}$. En gardant en tête que pour la rectification finale - conclusion - il faut encore «extraire la racine». Dans tous les cas c'est ici le développement intellectuel qui distingue la première étape de la seconde.

\section{Traduction du russe: Antoine Cattin, Valérie Posener Notes: François Albera, Antoine Cattin, Valérie Posener}

\section{NOTES DE L'ÉDITEUR}

1. "A. I." (en russe "I. A.») pour: Attraction intellectuelle. La notion avait été avancée dans quelques articles contemporains comme "Perspectives", "Notre Octobre. Au-delà du joué et du non-joué " et ultérieurs comme "Hors-Cadre" et "Dramaturgie der Film-Form" [Stuttgart]. Une première publication en russe figure dans l'ouvrage de R. Iourenev, Sergueï Eisenstein. Zamysly. Fil'my. Metoda (Tome I [18981929]. Moscou: Iskousstvo, 1985, p. 235-239) avec la page 16 en fac-similé reprise et traduite dans F. Albera, Eisenstein et le constructivisme russe (Lausanne: L'Âge d'Homme, 1990, p. 204). La présente version se base sur l'édition russe de la revue Kinoviedtcheuskie zapiski établie d'après le manuscrit de RGALI (op. 1, ed. chr. 980, Il. 1-20, p. 38-52). La confrontation avec le fac-similé évoqué ci-dessus laisse cependant apparaitre plusieurs altérations dans l'ordre du texte et dans la restitution des mots (par ex. on transcrit le français "sauf les lions!!!" en : "saut de lion!»).

2. Henri Bergson, Le Rire (Paris: Presses universitaires de France, 1953, p. 34). On trouve la même citation dans un texte contemporain de V. Chklovski, Sur les traces $d u$ marquis (Moscou: 1927).

3. En russe, losung.

4. En russe, resolutsiza.

5. Eisenstein désigne plus précisément ici la secte des flagellants.

Inédit : A.I. 28 [Attraction intellectuelle 1928] 
6. Euvres respectivement de Karamzine et de Hasek.

7. Posredrabisnik, acteur enregistré au «Posredrabis", bureau de recrutement des acteurs et autres travailleurs du théâtre.

8. Allusion au film de Jakob Protazanov, Don Diego et Pélagie (1927), représentant l'ancienne école d'acteurs et de mise en scène russe.

9. Obrazovannost', éducation, niveau culturel, comprend le mot abraz, image.

10. Ioffé (Abraham Moisevitch Deborine) (1891-1963), militant révolutionnaire, responsable de la revue Sous la bannière du marxisme en 1922, enseignant et chercheur en philosophie, comme représentant des "dialecticiens", il sera en butte, par la suite, à l'attaque des « mécanistes».

11. 1905 (désigné aussi par l'expression «la Cinquième " [année]) est le titre du film qu'Eisenstein commença de tourner d'après le scénario de N. Agadjanova-Choutko avant de l'abandonner pour le seul épisode du Cuirassé Potemkine. C'est à ce film inachevé dont certaines photographies étaient parues dans la presse que fait allusion Malévitch dans ses articles sur le cinéma.

12. Il s'agit de la sculpture de Rodin auprès de laquelle se trouvent les soldates du bataillon féminin, dépenaillées et vulgaires.

13. Le mot russe "mat" " (la mère) est l'homonyme du mot "mat", argot; "maternité " peut donc se lire également "argotisme ". Dans le passage en question d'Octobre, un soldat qui a quitté son poste se fait insulter par un officier et l'intertitre indique: - Votre mère! Le montage enchaîne ensuite sur l'inscription gravée sur le socle d'une statue: "Les premiers pas. Mère et enfant", puis on voit les personnages de la sculpture, une mère apprenant à marcher à son enfant, un gros plan de face de l'enfant puis un plan où une femme-officier commande l'exercice d'assaut de trois soldates dont les crosses de fusil obstruent latéralement, d'avant en arrière, le groupe sculptural.

14. Une expression injurieuse comportant le mot "mère" correspondant à l'expression "Nique ta mère!" en français contemporain.

15. Il s'agit de la suite intitulée parfois "la polonaise des dieux " qu'Eisenstein a souvent citée et commentée (par exemple dans "Dramaturgie der Film-Form»; voir également l'une des lettres à Moussinac ci-après).

16. Der letze Mann (1925) de Friedrich-Wilhelm Murnau.

17. Le livre de A. Belenson, Kino sevodnya. Vertov, Koulechov, Eisenstein ([Le Cinéma aujourd'hui. Kouléchov - Vertov - Eisenstein] Moscou: Teakinopetchat, 1924) dont le sous-titre était Aperçu de l'art cinématographique soviétique, publia une version altérée du premier texte théorique d'Eisenstein, "Le montage des attractions au cinéma ". 